International Electronic Journal of Geometry

Volume 8 No. 2 PP. 46-56 (2015) C)IEJG

\title{
HEMI-SLANT SUBMANIFOLDS OF A LOCALLY CONFORMAL KÄHLER MANIFOLD
}

\author{
HAKAN METE TAŞTAN AND SIBEL GERDAN
}

(Communicated by Levent KULA)

\begin{abstract}
In the present paper, we study hemi-slant submanifolds of a locally conformal Kähler manifold. We give conditions for the integrability of antiinvariant and slant distributions which are involved in the definition of hemislant submanifold. We also get necessary and sufficient conditions for these distributions to define totally geodesic foliations. The paper ends with some results for hemi-slant submanifolds with parallel canonical structures.
\end{abstract}

\section{INTRODUCTION}

Study of slant submanifolds was initiated by B.Y. Chen [4], as a generalization of both holomorphic and totally real submanifolds of a Kähler manifold. Slant submanifolds have been studied in different kinds of structures of both almost Hermitian and almost contact manifolds by several geometers. N. Papaghiuc [6] introduced semi-slant submanifolds of a Kähler manifold as a natural generalization of slant submanifold. These types of submanifolds have been studied in different types of structures, for example, see [7] and [12]. On the other hand, A. Carriazo [2], introduced the notion of bi-slant submanifold as a generalization of a semislant submanifold. One of the classes of bi-slant submanifolds is that of anti-slant submanifolds which are studied by A. Carriazo [2], but B. Şahin [9] named these submanifolds as hemi-slant submanifolds because of that the name anti-slant seems to refer that they have no slant factor. It is also seen that a hemi-slant submanifold is a special case of generic submanifold which was introduced by G.S. Ronsse [8]. Since then many geometers have studied hemi-slant submanifolds in different kinds of structures. For example, see [1] and [13]. In the present paper, we study hemislant submanifolds of a locally conformal Kähler manifold.

The paper is organized as follows. Section 2 is devoted to preliminaries. Actually, in subsection 2.1 we present the basic background needed for a locally conformal Kähler manifold. Theory of submanifolds and distributions needed for the study are placed in subsection 2.2. In subsection 2.3, we define hemi-slant submanifolds and

Date: Received: January 19, 2015 and Accepted: August 12, 2015.

2010 Mathematics Subject Classification. Primary 53B25; Secondary 53C55.

Key words and phrases. Locally conformal Kähler manifold, hemi-slant submanifold, slant distribution. 
observe their effect to the tangent bundle of the submanifold and canonical projection operators. We begin to study hemi-slant submanifolds of a locally conformal Kähler manifold in section 3. Integrability of anti-invariant and slant distributions of the hemi-slant submanifold are studied in this section. We also give necessary and sufficient conditions for these distributions to define totally geodesic foliations in section 3. The last section deals with parallelism of the canonical projection structures on the tangent bundle of the hemi-slant submanifold.

\section{PRELiminaries}

2.1. Some background for a locally conformal Kähler manifold. A smooth manifold $\bar{M}$ is called almost Hermitian [15] if its tangent bundle has an almost complex structure $J$ and a Riemannian metric $g$ such that

$$
g(\bar{U}, \bar{V})=g(J \bar{U}, J \bar{V})
$$

for any vector fields $\bar{U}, \bar{V} \in T \bar{M}$, where $T \bar{M}$ is the tangent bundle of $\bar{M}$. The manifold $(\bar{M}, g, J)$ is called a locally conformal Kähler manifold (briefly l.c.K. manifold), if every point of $\bar{M}$ has a neighborhood $\mathcal{U}$ such that the restriction $\left.g\right|_{\mathcal{U}}$ of $g$ to $\mathcal{U}$ is conformal to a Kähler metric $g_{\mathcal{U}}^{\prime}$ of $\mathcal{U}: g_{\mathcal{U}}^{\prime}=\left.e^{-\sigma} g\right|_{\mathcal{U}}$ for some $C^{\infty}$-function $\sigma: \mathcal{U} \rightarrow \mathbb{R} .(\bar{M}, g)$ is a globally conformal Kähler manifold (briefly g.c.K. manifold) if one can choose $\mathcal{U}=\bar{M}$; then $g^{\prime}$ is a Kähler metric on $\bar{M}$, and hence $\left(\bar{M}, g^{\prime}\right)$ is a Kähler manifold.

Let $\Omega$ be 2 -form on $\bar{M}$. Then $\bar{M}$ is a l.c.K. manifold if and only if there is a global 1-form $\omega$ on $\bar{M}$ (the Lee form of $\bar{M}$ ) such that [14]

$$
d \Omega=\omega \wedge \Omega, \quad d \omega=0
$$

and $\bar{M}$ is a g.c.K. manifold if $\omega$ is also exact. For a l.c.K. manifold the Lee vector field $B$ is given by

$$
g(B, \bar{U})=\omega(\bar{U})
$$

for any vector field $\bar{U}$ on $\bar{M}$. We denote by $\tilde{\nabla}$ (resp. $\bar{\nabla}$ ) is the Riemannian connection of $\left.e^{-\sigma} g\right|_{\mathcal{U}}($ resp. $g$ ). Then we have [14]

$$
\tilde{\nabla}_{\bar{U}} \bar{V}=\bar{\nabla}_{\bar{U}} \bar{V}-\frac{1}{2}\{\omega(\bar{U}) \bar{V}+\omega(\bar{V}) \bar{U}-g(\bar{U}, \bar{V}) B\}
$$

for any vector fields $\bar{U}, \bar{V}$ on $\bar{M}$. The connection $\tilde{\nabla}$ is a torsionless linear connection on $\bar{M}$ which is called the Weyl connection of $g$. It is easy to see that the Weyl connection $\tilde{\nabla}$ satisfies the condition

$$
\tilde{\nabla} J=0 .
$$

For more details on l.c.K. manifolds we refer to [5].

2.2. Submanifolds. Let $M$ be a submanifold of a Riemannian manifold $\bar{M}$ with a Riemannian metric $g$. Then Gauss and Weingarten formulas with respect to $\bar{\nabla}$ are given respectively by

$$
\bar{\nabla}_{U} V=\nabla_{U} V+h(U, V)
$$

and

$$
\bar{\nabla}_{U} \xi=-A_{\xi} U+\nabla_{U}^{\perp} \xi
$$


for all $U, V \in T M$ and $\xi \in T^{\perp} M$, where and $\nabla$ and $\nabla^{\perp}$ are respectively the induced Riemannian, and induced normal connection with respect to $\bar{\nabla}$ in $M$ and the normal bundle $T^{\perp} M$ of $M$ and $h$ is the second fundamental form related to shape operator $A$ corresponding to the normal vector field $\xi$ by

$$
g(h(U, V), \xi)=g\left(A_{\xi} U, V\right) .
$$

A submanifold $M$ is said to be totally geodesic if its second fundamental form vanishes identically, that is, $h=0$, or equivalently $A_{\xi}=0$. We say that $M$ is totally umbilical submanifold in $\bar{M}$ if for all $U, V \in T M$ we have

$$
h(U, V)=g(U, V) H,
$$

where $H$ is the mean curvature vector field of $M$ in $\bar{M}$.

Let $M$ be a submanifold of an almost Hermitian manifold $(\bar{M}, g, J)$. For any $U \in T M$ we write

$$
J U=P U+F U,
$$

where $P U$ is the tangential part of $J U$, and $F U$ is the normal part of $J U$. Similarly, for any $\xi \in T^{\perp} M$, we put

$$
J \xi=t \xi+f \xi
$$

where $t \xi$ is the tangential part of $J \xi$, and $f \xi$ is the normal part of $J \xi$.

A distribution $\mathcal{D}$ on a manifold $\bar{M}$ is called autoparallel if $\nabla_{X} Y \in \mathcal{D}$ for any $X, Y \in \mathcal{D}$ and called parallel if $\bar{\nabla}_{U} X \in \mathcal{D}$ for any $X \in \mathcal{D}$ and $U \in T \bar{M}$. If a distribution $\mathcal{D}$ on $\bar{M}$ is autoparallel, then it is clearly integrable, and by Gauss formula $\mathcal{D}$ is totally geodesic in $\bar{M}$. If $\mathcal{D}$ is parallel then the orthogonal complementary distribution $\mathcal{D}^{\perp}$ is also parallel, which implies that $\mathcal{D}$ is parallel if and only if $\mathcal{D}^{\perp}$ is parallel. In this case $\bar{M}$ is locally product of the leaves of $\mathcal{D}$ and $\mathcal{D}^{\perp}$. For two distributions $\mathcal{D}_{1}$ and $\mathcal{D}_{2}$ on a submanifold $M$ of $\bar{M}$, we say that $M$ is $\left(\mathcal{D}_{1}, \mathcal{D}_{2}\right)$ mixed totally geodesic, if for all $X \in \mathcal{D}_{1}$ and $Y \in \mathcal{D}_{2}$ we have $h(X, Y)=0$, where $h$ is the second fundamental form of $M[11,15]$.

2.3. Hemi-slant submanifolds of an almost Hermitian manifold. Let $M$ be a submanifold of $\bar{M}$. A distribution $\mathcal{D}$ on $M$ is said to be a slant distribution if for $X \in \mathcal{D}_{p}$, the angle $\theta$ between $J X$ and $\mathcal{D}_{p}$ is constant, i.e., independent of $p \in M$ and $X \in \mathcal{D}_{p}$. The constant angle $\theta$ is called the slant angle of the slant distribution $\mathcal{D}$. It is well-known that holomorphic and totally real distributions on $M$ are slant distributions with $\theta=0$ and $\theta=\frac{\pi}{2}$, respectively. A submanifold $M$ of $\bar{M}$ is said to be a slant submanifold if the tangent bundle $T M$ of $M$ is slant [4].

A hemi-slant submanifold $[2,9] M$ of an almost Hermitian manifold $\bar{M}$ is a submanifold which admits two orthogonal complementary distributions $\mathcal{D}^{\perp}$ and $\mathcal{D}^{\theta}$ such that

(a) $T M$ admits the orthogonal direct decomposition $T M=\mathcal{D}^{\perp} \oplus \mathcal{D}^{\theta}$

(b) The distribution $\mathcal{D}^{\perp}$ is anti-invariant, i.e., $J \mathcal{D}^{\perp} \subseteq T^{\perp} M$. 
(c) The distribution $\mathcal{D}^{\theta}$ is slant with slant angle $\theta$.

We say that the hemi-slant submanifold $M$ is proper if $d \neq 0$ and $\theta \neq 0, \frac{\pi}{2}$, where $d$ denotes the dimension of $\mathcal{D}^{\perp}$. In this case, we have

$$
\begin{gathered}
P^{2} Z=-\cos ^{2} \theta Z, \\
g(P Z, P W)=\cos ^{2} \theta g(Z, W)
\end{gathered}
$$

and

$$
g(F Z, F W)=\sin ^{2} \theta g(Z, W)
$$

for any $Z, W \in \mathcal{D}^{\theta}[9]$.

Lemma 2.1. Let $M$ be a proper hemi-slant submanifold of an almost Hermitian manifold $\bar{M}$. Then we have,

$$
J \mathcal{D}^{\perp} \perp F \mathcal{D}^{\theta}
$$

Proof. For any $X \in \mathcal{D}^{\perp}$ and $Z \in \mathcal{D}^{\theta}$, using (2.1) and (2.9), we have $g(J X, F Z)=g(J X, J Z)=g(X, Z)=0$. This completes the proof.

In view of Lemma 2.1, for a hemi-slant submanifold $M$ of a l.p.R. manifold $\bar{M}$, the normal bundle $T^{\perp} M$ of $M$ is decomposed as

$$
T^{\perp} M=F \mathcal{D}^{\theta} \oplus J \mathcal{D}^{\perp} \oplus \mu,
$$

where $\mu$ is the orthogonal complementary distribution of $F \mathcal{D}^{\theta} \oplus J \mathcal{D}^{\perp}$ in $T^{\perp} M$ and it is invariant subbundle of $T^{\perp} M$ with respect to $J$. Using (2.9), (2.10) and the fact that $J^{2}=-I$, we have the following facts.

$$
\begin{aligned}
& \text { (a) } P^{2}+t F=-I \text {, } \\
& \text { (b) } f^{2}+F t=-I \text {, } \\
& \text { (c) } F P+f F=0 \text {, } \\
& \text { (d) } t f+P t=0 \text {. }
\end{aligned}
$$

Lemma 2.2. Let $M$ be a proper hemi-slant submanifold of an almost Hermitian manifold $\bar{M}$. Then we have,

$$
\text { (a) } P \mathcal{D}^{\perp}=\{0\}, \quad \text { (b) } P \mathcal{D}^{\theta}=\mathcal{D}^{\theta}
$$

Proof. Since $\mathcal{D}^{\perp}$ is anti-invariant with respect to $J$, (a) follows from (2.9). For any $Z \in \mathcal{D}^{\theta}$ and $X \in \mathcal{D}^{\perp}$, using (2.1) and (2.9), we have $g(P Z, X)=g(J Z, X)=$ $g(Z, J X)=0$. Hence, we conclude that $P \mathcal{D}^{\theta} \perp \mathcal{D}^{\perp}$. Since $P \mathcal{D}^{\theta} \subseteq T M$, it follows that $P \mathcal{D}^{\theta} \subseteq \mathcal{D}^{\theta}$. Let $W$ be in $\mathcal{D}^{\theta}$. Then using (2.11), we have $W=\frac{1}{\cos ^{2} \theta}\left(\cos ^{2} \theta W\right)=$ $\frac{-1}{\cos ^{2} \theta} P^{2} W=\frac{-1}{\cos ^{2} \theta} P(P W)$. So, we find $W \in P \mathcal{D}^{\theta}$. It follows that $\mathcal{D}^{\theta} \subseteq P \mathcal{D}^{\theta}$. Thus, we get the assertion (b).

\section{Hemi-Slant SUBmanifolds of A LOCALly CONFORMAL KäHLER MANifOld}

Let $M$ be a (proper) hemi-slant submanifold of a l.c.K. manifold $\bar{M}$ with antiinvariant distribution $\mathcal{D}^{\perp}$ and slant distribution $\mathcal{D}^{\theta}$. For the Lee vector field $B$ of $\bar{M}$, we put

$$
B=B^{T}+B^{N} \quad(\text { along } \quad M),
$$

where $B^{T}$ and $B^{N}$ are respectively tangential and normal part of $B$.

First, we give an example. 
Example 3.1. Consider the almost Hermitian manifold $\left(\mathbb{R}^{6} \backslash\{0\}, g, J\right)$,

where $g=\lambda^{-1}\left(d x_{1}^{2}+\ldots+d x_{6}^{2}\right),\left(\lambda=x_{1}^{2}+\ldots+x_{6}^{2}\right)$ is a Riemannian metric and the almost complex structure $J$ defined by

$$
J \partial_{1}=\partial_{2}, J \partial_{2}=-\partial_{1}, \ldots, J \partial_{5}=\partial_{6}, J \partial_{6}=-\partial_{5}
$$

Finally, consider on $\mathbb{R}^{6} \backslash\{0\}$, the Riemannian metric $\tilde{g}=\lambda g$. Then it is easy to see that $\left(\mathbb{R}^{6} \backslash\{0\}, \tilde{g}, J\right)$ is a Kähler manifold. So, $\left(\mathbb{R}^{6} \backslash\{0\}, g, J\right)$ is a globally conformal Kähler manifold with the Lee form

$$
\omega=-2 \lambda^{-1}\left(x_{1} d x_{1}+\ldots+x_{6} d x_{6}\right) .
$$

Consequently, the Lee vector field

$$
B=-2 \lambda^{-1}\left(x_{1} \partial_{1}+\ldots+x_{6} \partial_{6}\right) .
$$

Now, let $M$ be a submanifold of $\left(\mathbb{R}^{6} \backslash\{0\}, g, J\right)$ defined by

$$
f(u, v, w)=\left(\frac{u}{\sqrt{2}} \cos v, \frac{u}{\sqrt{2}} \sin v, \frac{u}{\sqrt{2}}, 0, w, 0\right)
$$

where $u, w \neq 0$. Then, a local frame of $T M$ is given by

$$
\begin{aligned}
& Z=\frac{1}{\sqrt{2}}\left(\cos v \partial_{1}+\sin v \partial_{2}+\partial_{3}\right), \\
& W=\frac{u}{\sqrt{2}}\left(-\sin v \partial_{1}+\cos v \partial_{2}\right), \\
& X=\partial_{5} .
\end{aligned}
$$

By using the almost complex structure $J$ and the Riemannian metric $g$ above, we see that $J X$ is orthogonal to $T M$, thus $\mathcal{D}^{\perp}=\operatorname{span}\{\mathrm{X}\}$. Moreover, it is not difficult to see that $\mathcal{D}^{\theta}=\operatorname{span}\{Z, W\}$ is a slant distribution with slant angle $\theta=\pi / 4$. Thus, $M$ is a proper hemi-slant submanifold of $\left(\mathbb{R}^{6} \backslash\{0\}, g, J\right)$.

Now, we study the integrability of anti-invariant distribution $\mathcal{D}^{\perp}$ and slant distribution $\mathcal{D}^{\theta}$.

Lemma 3.1. Let $M$ be any submanifold of a l.c.K. manifold $\bar{M}$. Then we have

$$
\begin{aligned}
& \nabla_{U} P V-A_{F V} U-\frac{1}{2} \omega(J V) U+\frac{1}{2} g(U, P V) B^{T} \\
& =P \nabla_{U} V+t h(U, V)-\frac{1}{2} \omega(V) P U+\frac{1}{2} g(U, V)\left(P B^{T}+t B^{N}\right)
\end{aligned}
$$

and

$$
\begin{aligned}
& \nabla_{U}^{\perp} F V+h(U, P V)+\frac{1}{2} g(U, P V) B^{N} \\
& =F \nabla_{U} V+f h(U, V)-\frac{1}{2} \omega(V) F U+\frac{1}{2} g(U, V)\left(F B^{T}+f B^{N}\right)
\end{aligned}
$$

for any $U, V \in T M$.

Proof. Substituting $V$ by $J V$ in (2.4) and using (2.9) and (2.5) we obtain

$$
\begin{aligned}
& \nabla_{U} P V+h(U, P V)+\nabla_{U}^{\perp} F V-A_{F V} U \\
- & \frac{1}{2} \omega(U) J V-\frac{1}{2} \omega(J V) U+\frac{1}{2} g(U, J V) B \\
= & P \nabla_{U} V+F \nabla_{U} V+t h(U, V)+f h(U, V) \\
- & \frac{1}{2} \omega(U) J V-\frac{1}{2} \omega(V) J U+\frac{1}{2} g(U, V) J B
\end{aligned}
$$


Thus, (3.2) and (3.3) follow from (3.4) by using (2.10), (2.11) and identifying the components from $T M$ and $T^{\perp} M$.

Proposition 3.1. Let $M$ be a hemi-slant submanifold of a l.c.K. manifold $\bar{M}$. Then the anti-invariant distribution $\mathcal{D}^{\perp}$ is integrable if and only if

$$
A_{F X} Y+\frac{1}{2} \omega(F X) Y=A_{F Y} X+\frac{1}{2} \omega(F Y) X
$$

for any $X, Y \in \mathcal{D}^{\perp}$.

Proof. Let $X, Y$ be in $\mathcal{D}^{\perp}$. Then using (2.17)-(a), we have

$$
-A_{F Y} X-\frac{1}{2} \omega(F Y) X=P \nabla_{X} Y+t h(X, Y)+\frac{1}{2} g(X, Y)\left(P B^{T}+t B^{N}\right)
$$

from (3.2). Since $h$ and $g$ are symmetric $(0,2)$-type of tensor fields, it follows from (3.6) that

$$
P[X, Y]=A_{F X} Y-A_{F Y} X+\frac{1}{2}\{\omega(F X) Y-\omega(F Y) X\} .
$$

With the help of (2.17)-(a), we see that $\mathcal{D}^{\perp}$ is integrable if and only if $P[X, Y]=0$ for $X, Y \in \mathcal{D}^{\perp}$. So, our assertion follows easily from (3.7).

Proposition 3.2. Let $M$ be a hemi-slant submanifold of a l.c.K. manifold $\bar{M}$. Then the slant distribution $\mathcal{D}^{\theta}$ is integrable if and only if

$$
\nabla_{Z} P W-\nabla_{W} P Z+A_{F Z} W-A_{F W} Z+g(Z, P W) B^{T} \in \mathcal{D}^{\theta}
$$

for any $Z, W \in \mathcal{D}^{\theta}$.

Proof. The slant distribution $\mathcal{D}^{\theta}$ is integrable if and only if $[Z, W] \in \mathcal{D}^{\theta}$, for any $Z, W \in \mathcal{D}^{\theta}$. On the other hand $[Z, W] \in \mathcal{D}^{\theta}$ if and only if $P[Z, W] \in \mathcal{D}^{\theta}$, because of (2.17)-(b) and the fact that $T M=\mathcal{D}^{\perp} \oplus \mathcal{D}^{\theta}$. For any $Z, W \in \mathcal{D}^{\theta}$, using (2.10), we have

$$
\begin{aligned}
& \nabla_{Z} P W-A_{F W} Z-\frac{1}{2} \omega(J W) Z+\frac{1}{2} g(Z, P W) B^{T} \\
& =P \nabla_{Z} W+t h(Z, W)-\frac{1}{2} \omega(W) P Z+\frac{1}{2} g(Z, W)\left(P B^{T}+t B^{N}\right)
\end{aligned}
$$

from (3.2). Since $h$ and $g$ are symmetric $(0,2)$-type of tensor fields, it follows from (3.9) that

$$
\begin{aligned}
P[Z, W] & =\nabla_{Z} P W-\nabla_{W} P Z+A_{F Z} W-A_{F W} Z+g(Z, P W) B^{T} \\
& -\frac{1}{2} \omega(J W) Z+\frac{1}{2} \omega(J Z) W+\frac{1}{2} \omega(W) P Z-\frac{1}{2} \omega(Z) P W .
\end{aligned}
$$

Using (2.17)-(b) from (3.10), we find $P[Z, W] \in \mathcal{D}^{\theta}$ if and only if (3.8) holds.

We also proved the Lemma 3.1 and Proposition 3.2 for semi-slant submanifolds of a l.c.K. manifold in [12].

Let $M$ be a hemi-slant submanifold of a l.c.K. manifold $\bar{M}$. Then Gauss and Weingarten formulas with respect to $\tilde{\nabla}$ are given respectively by

$$
\tilde{\nabla}_{U} V=\hat{\nabla}_{U} V+\tilde{h}(U, V),
$$

and

$$
\tilde{\nabla}_{U} \xi=-\tilde{A}_{\xi} U+\tilde{\nabla}_{U}^{\perp} \xi
$$

for all $U, V \in T M$ and $\xi \in T^{\perp} M$, where $\hat{\nabla}$ and $\tilde{\nabla}^{\perp}$ are respectively the induced Riemannian and induced normal connection with respect to $\tilde{\nabla}$ in $M$ and the normal 
bundle $T^{\perp} M$ of $M$, and $\tilde{h}$ is the second fundamental form of $M$ with respect to $\tilde{\nabla}$. Moreover, the second fundamental form $\tilde{h}$ is related to the shape operator $\tilde{A}$ by

$$
g(\tilde{h}(U, V), \xi)=g\left(\tilde{A}_{\xi} U, V\right) .
$$

Using (2.5), (2.6), (3.11) and (3.12) from (2.3) we have the following lemma.

Lemma 3.2. Let $M$ be a hemi-slant submanifold of a l.c.K. manifold $\bar{M}$. Then we have

$$
\begin{gathered}
\hat{\nabla}_{U} V=\nabla_{U} V-\frac{1}{2}\left\{\omega(U) V+\omega(V) U-g(U, V) B^{T}\right\}, \\
\tilde{h}(U, V)=h(U, V)+\frac{1}{2} g(U, V) B^{N}, \\
\tilde{A}_{\xi} U=A_{\xi} U+\frac{1}{2} \omega(\xi) U, \\
\tilde{\nabla}_{U}^{\perp} \xi=\nabla_{U}^{\perp} \xi-\frac{1}{2} \omega(U) \xi
\end{gathered}
$$

for any $U, V \in T M$ and $\xi \in T^{\perp} M$.

We remark that Lemma 3.2 was also given as Lemma 2.1 in ([10]) for generic submanifolds (in the sense of Chen [3]) of a l.c.K. manifold.

From Proposition 3.1 and (3.16), we have the following result.

Corollary 3.1. Let $M$ be a proper hemi-slant submanifold of a l.c.K. manifold $\bar{M}$. Then the anti-invariant distribution $\mathcal{D}^{\perp}$ is integrable if and only if

$$
\tilde{A}_{F X} Y=\tilde{A}_{F Y} X
$$

for any $X, Y \in \mathcal{D}^{\perp}$.

Lemma 3.3. Let $M$ be a hemi-slant submanifold of a l.c.K. manifold $\bar{M}$. Then we have

$$
-P\left(\hat{\nabla}_{U} X\right)=\tilde{A}_{F X} U+t \tilde{h}(U, X)
$$

for any $X \in \mathcal{D}^{\perp}$ and $U \in T M$.

Proof. From (2.5), we have $\tilde{\nabla}_{U} J X=J \tilde{\nabla}_{U} X$ for any $X \in \mathcal{D}^{\perp}$ and $U \in T M$. Using (2.17)-(a) and (3.11), we obtain

$$
\tilde{\nabla}_{U} F X=J\left(\hat{\nabla}_{U} X\right)+J \tilde{h}(U, X) .
$$

Hence, it follows that

$$
-\tilde{A}_{F X} U+\tilde{\nabla}_{U}^{\perp} F X=P\left(\hat{\nabla}_{U} X\right)+F\left(\hat{\nabla}_{U} X\right)+t \tilde{h}(U, X)+f \tilde{h}(U, X) .
$$

Taking the tangential part of this equation we find (3.19).

Theorem 3.1. Let $M$ be a proper hemi-slant submanifold of a l.c.K. manifold $\bar{M}$. Then the anti-invariant distribution $\mathcal{D}^{\perp}$ is integrable.

Proof. With the help of (2.17)-(a) and (2.17)-(b), for any $X, Y \in \mathcal{D}^{\perp}$ and $U \in T M$, we have

$0=g\left(-P\left(\hat{\nabla}_{U} X\right), Y\right)=g\left(\tilde{A}_{F X} U, Y\right)+g(t \tilde{h}(U, X), Y)$ from Lemma 3.3. After some calculation, we find $g\left(\tilde{A}_{F X} Y, U\right)=g\left(\tilde{A}_{F Y} X, U\right)$. It follows that $\tilde{A}_{F X} Y=$ $\tilde{A}_{F Y} X$. Thus our assertion follows from Corollary 3.1. 
Next, we are going to study the problem when a proper hemi-slant of submanifold of a l.c.K. manifold is a Riemannian product of a totally real submanifold and a slant submanifold.

Theorem 3.2. Let $M$ be a proper hemi-slant submanifold of a l.c.K. manifold $\bar{M}$. Then the slant distribution $\mathcal{D}^{\theta}$ defines a totally geodesic foliation on $M$ if and only if

$$
g\left(A_{F X} W+\frac{1}{2} \omega(F X) W, Z\right)=g\left(A_{F W} X, Z\right)
$$

for all $Z, W \in \mathcal{D}^{\theta}$ and $X \in \mathcal{D}^{\perp}$.

Proof. We know that $\left(\bar{M}, g^{\prime}, J\right)$ is a Kähler manifold. Thus, from Theorem 3.7 [9], we have the distribution $\mathcal{D}^{\theta}$ defines a totally geodesic foliation on $M$ if and only if

$$
g^{\prime}\left(\tilde{A}_{F X} P W, Z\right)=g^{\prime}\left(\tilde{A}_{F P W} X, Z\right)
$$

for all $Z, W \in \mathcal{D}^{\theta}$ and $X \in \mathcal{D}^{\perp}$. Putting $W=P W$ in this equation and using (2.11), we get

$$
g^{\prime}\left(\tilde{A}_{F X} W, Z\right)=g^{\prime}\left(\tilde{A}_{F W} X, Z\right) .
$$

Thus, our assertion follows from (3.16), (3.21) and the fact that $g(X, Z)=0$.

Theorem 3.3. Let $M$ be a proper hemi-slant submanifold of a l.c.K. manifold $\bar{M}$. Then the anti-invariant distribution $\mathcal{D}^{\perp}$ defines a totally geodesic foliation on $M$ if and only if

$$
g\left(A_{F X} Z, Y\right)=g\left(A_{F Z} X+\frac{1}{2} \omega(F Z) X, Y\right)
$$

for all $X, Y \in \mathcal{D}^{\perp}$ and $Z \in \mathcal{D}^{\theta}$.

Proof. Since $\left(\bar{M}, g^{\prime}, J\right)$ is a Kähler manifold, it follows from Theorem 3.8 [9] that the distribution $\mathcal{D}^{\perp}$ defines a totally geodesic foliation on $M$ if and only if

$$
g^{\prime}\left(\tilde{A}_{F X} P Z, Y\right)=g^{\prime}\left(\tilde{A}_{F P Z} X, Y\right)
$$

for all $X, Y \in \mathcal{D}^{\perp}$ and $Z \in \mathcal{D}^{\theta}$. Putting $Z=P Z$ in this equation and using (2.11), we get

$$
g^{\prime}\left(\tilde{A}_{F X} Z, Y\right)=g^{\prime}\left(\tilde{A}_{F Z} X, Y\right) .
$$

Thus, our assertion follows from (3.16), (3.23) and the fact that $g(Y, Z)=0$.

Corollary 3.2. Let $M$ be a proper hemi-slant submanifold of a l.c.K. manifold $\bar{M}$. Then $M$ is a locally Riemannian product manifold $M=M_{\mathcal{D}^{\perp}} \times M_{\mathcal{D}^{\theta}}$ if and only if

$$
A_{F X} Z+\frac{1}{2} \omega(F X) Z=A_{F Z} X+\frac{1}{2} \omega(F Z) X
$$

for $X \in \mathcal{D}^{\perp}$ and $Z \in \mathcal{D}^{\theta}$, where $M_{\mathcal{D}^{\perp}}$ is a totally real and $M_{\mathcal{D}^{\theta}}$ is a slant submanifold of $\bar{M}$.

Proof. It follows easily from (3.21), (3.23) and (3.16). 


\section{Hemi-SLANT SUBMANifOlds With PARALlEL CANONICAL STRUCTURES}

In this section, we study hemi-slant submanifolds of a l.c.K. manifold with parallel canonical projection structures on the tangent bundle of the submanifold.

Let $M$ be a submanifold of a l.c.K. manifold $\bar{M}$. For the endomorphism $P$ : $T M \rightarrow T M$ we put

$$
\left(\tilde{\nabla}_{U} P\right) V=\hat{\nabla}_{U} P V-P \hat{\nabla}_{U} V
$$

for any $U, V \in T M$. We say that $P$ is parallel if $\left(\tilde{\nabla}_{U} P\right)=0$ for any $U \in T M$. From (2.5), (3.11) and (3.12), we have

$$
\begin{aligned}
& \hat{\nabla}_{U} P V+\tilde{h}(U, P V)-\tilde{A}_{F V} U+\tilde{\nabla}_{U}^{\perp} F V \\
= & P \hat{\nabla}_{U} V+F \hat{\nabla}_{U} V+t \tilde{h}(U, V)+f \tilde{h}(U, V)
\end{aligned}
$$

Hence, we obtain

$$
\left(\tilde{\nabla}_{U} P\right) V=t \tilde{h}(U, V)+\tilde{A}_{F V} U
$$

Thus, for any $U, V, W \in T M$, we get

$$
g\left(\left(\tilde{\nabla}_{U} P\right) V, W\right)=g\left(\tilde{A}_{F V} W-\tilde{A}_{F W} V, U\right) .
$$

Proposition 4.1. Let $M$ be a submanifold of a l.c.K. manifold $\bar{M}$. Then $P$ is parallel if and only if

$$
A_{F U} V-A_{F V} U=\frac{1}{2}\{\omega(F V) U-\omega(F U) V\}
$$

for any $U, V \in T M$.

Proof. It follows immediately from (3.16) and (4.2).

Theorem 4.1. Let $M$ be a hemi-slant submanifold of a l.c.K. manifold $\bar{M}$. Then $P$ is parallel, i.e., $\tilde{\nabla} P \equiv 0$, if and only if the anti-invariant distribution $\mathcal{D}^{\perp}$ is autoparallel.

Proof. Let $P$ be parallel. For any $X, Y \in \mathcal{D}^{\perp}$, using (2.17)-(a), we have

$$
0=\left(\tilde{\nabla}_{X} P\right) Y=P \hat{\nabla}_{X} Y
$$

from (4.1). Again, using (2.17)-(a), we deduce that $\hat{\nabla}_{X} Y \in \mathcal{D}^{\perp}$ from (4.4). It means that $\mathcal{D}^{\perp}$ is autoparallel. Converse is clear.

Now, we give a characterization theorem for totally umbilical hemi-slant submanifolds of a l.c.K. manifold.

Theorem 4.2. Let $M$ be a totally umbilical hemi-slant submanifold of a l.c.K. manifold $\bar{M}$. If $P$ is parallel and $\operatorname{dim}\left(\mathcal{D}^{\perp}\right) \geq 2$, then the mean curvature vector field $\tilde{H}$ of $M$ belongs to distribution $\mu$.

Proof. Since $\operatorname{dim}\left(\mathcal{D}^{\theta}\right) \geq 2$, we choose $Z, W \in \mathcal{D}^{\theta}$ such that $g(Z, W)=0$ and $\|Z\|=1$. Then, using (2.4) and (3.13), we have

$$
g(\tilde{H}, F W)=g(\widetilde{h}(Z, Z), F W)=g\left(\tilde{A}_{F W} Z, Z\right) .
$$

Here, from Lemma 3.5 of $[4, p .23]$, we know that $\tilde{A}_{F W} Z=\tilde{A}_{F Z} W$. Thus, we get 


$$
\tilde{H} \perp F \mathcal{D}^{\theta} .
$$

On the other hand, we can choose $X, Y \in \mathcal{D}^{\perp}$ such that $g(X, Y)=0$ and $\|Y\|=1$ from the hypothesis. Again, using (2.5) and (3.13), we have

$g(\tilde{H}, F X)=g(\widetilde{h}(Y, Y), F X)=g\left(\tilde{A}_{F X} Y, Y\right)$. Here, by Theorem 4.1 and Corollary 3.1, we know that $\tilde{A}_{F X} Y=\tilde{A}_{F Y} X$. So, we get

$$
g(\tilde{H}, F X)=g\left(\tilde{A}_{F Y} X, Y\right)=g(\widetilde{h}(X, Y), F Y)=0 \text {. It follows that }
$$

$$
\tilde{H} \perp F \mathcal{D}^{\perp} \text {. }
$$

Thus, our assertion follows from (4.5), (4.6) and (2.15).

For the normal-bundle 1-form $F$, we put

$$
\left(\tilde{\nabla}_{U} F\right) V=\tilde{\nabla}_{U}^{\perp} F V-F \hat{\nabla}_{U} V .
$$

for any $U, V \in T M$. We say that $F$ is parallel if $\left(\tilde{\nabla}_{U} F\right)=0$ for any $U \in T M$. By using (3.11) and (3.12), for any $U, V \in T M$, we have

$$
\left(\tilde{\nabla}_{U} F\right) V=f \tilde{h}(U, V)-\tilde{h}(U, P V)
$$

from (4.7). Hence, for any $\xi \in T^{\perp} M$, we obtain

$$
\left.g\left(\tilde{\nabla}_{U} F\right) V, \xi\right)=-g\left(\tilde{A}_{f \xi} V+\tilde{A}_{\xi} P V, U\right) .
$$

for any $U, V \in T M$ and $\xi \in T^{\perp} M$.

Proposition 4.2. Let $M$ be a submanifold of a l.c.K. manifold $\bar{M}$. Then $F$ is parallel if and only if

$$
A_{f \xi} U+A_{\xi} P U=-\frac{1}{2}\{\omega(f \xi) U+\omega(\xi) P U\}
$$

for any $U \in T M$ and $\xi \in T^{\perp} M$.

Proof. It follows immediately from (3.16) and (4.9).

Theorem 4.3. Let $M$ be a proper hemi-slant submanifold of a l.c.K. manifold $\bar{M}$. If $F$ is parallel, then $M$ is mixed totally geodesic.

Proof. Let $F$ be parallel. Then, from (4.8), we have

$$
f \tilde{h}(U, V)=\tilde{h}(U, P V) .
$$

for any $U, V \in T M$. Hence, we obtain

$$
f^{2} \tilde{h}(U, V)=f \tilde{h}(U, P V)=\tilde{h}\left(U, P^{2} V\right) .
$$

In particular, if we put $V=X \in \mathcal{D}^{\perp}$ and $U=Z \in \mathcal{D}^{\theta}$ in (4.11), with the help of (2.17)-(a), we obtain

$$
f^{2} \tilde{h}(Z, X)=\tilde{h}\left(Z, P^{2} X\right)=0 .
$$

On the other hand, using (2.11), we have

$$
f^{2} \tilde{h}(Z, X)=f^{2} \tilde{h}(X, Z)=\tilde{h}\left(X, P^{2} Z\right)=-\cos ^{2} \theta \tilde{h}(X, Z)
$$

from (4.11). Since $\theta \neq \frac{\pi}{2}$, for any $X \in \mathcal{D}^{\perp}$ and $Z \in \mathcal{D}^{\theta}$, we deduce that

$$
\tilde{h}(X, Z)=0
$$


from (4.12) and (4.13). Thus, our assertion easily comes from (3.15) and (4.14).

\section{REFERENCES}

[1] Al-Solamy, F.R., Khan M.A. and Uddin, S., Totally umbilical hemi-slant submanifolds of Kähler manifolds, Abstr. Appl. Anal. 2011, Art. ID 987157, 9 pp.

[2] Carriazo, A., Bi-slant immersions, in:Proc. ICRAMS 2000, Kharagpur,India, 2000, 88-97.

[3] Chen, B.-Y., Differential geometry of real submanifolds in a Kaehler manifold, Monatsh Math. 91(1981), 257-274

[4] Chen, B.-Y., Geometry of slant submanifolds, Katholieke Universiteit Leuven, 1990.

[5] Dragomir, S. and Ornea, L., Locally conformal Kähler geometry, Progress in Mathematicsi 155.Birkhäuser Boston, Inc., Boston, MA, 1998.

[6] Papaghiuc, N., Semi-slant submanifolds of a Kählerian manifold, Ann. Şt. Al. I. Cuza Univ. Iaşi 40(1994), 55-61.

[7] Li, H. and Liu, X., Semi-slant submanifolds of a locally product manifold, Georgian Math. J. 12(2005), no. 2, 273-282.

[8] Ronsse, G.S., Generic and skew $C R$-submanifolds of a Kähler manifold, Bull. Inst. Math. Acad. Sinica 18(1990), 127-141.

[9] Şahin, B., Warped product submanifolds of a Kähler manifold with a slant factor, Ann. Pol. Math. 95(2009), no. 3, 207-226.

[10] Shahid, M.H. and Husain, S.I., Generic submanifolds of a locally conformal Kaehler manifold, Soochow J. of Math. 14(1988), no. 1, 111-117.

[11] Tripathi, M.M., On $C R$ submanifolds of nearly and closely cosympletic manifolds, Ganita, $51(2000)$, no. $1,45-56$.

[12] Taştan, H.M. and Tripathi, M.M., Semi-slant submanifolds of a locally conformal Kähler manifold, Ann. Şt. Al. I. Cuza Univ. Iaşi, 2014 (accepted).

[13] Uddin, S., Khan M.A. and Singh, K., A note on totally umbilical pseudo-slant submanifolds of a nearly Kähler manifold, Acta Univ. Apulensis Math. Inform. No. 29(2012), 279-285.

[14] Vaisman, I., Some curvature properties of locally conformal Kähler manifolds, Trans. Amer. Math. Soc. 259(1980), no. 2, 439-447.

[15] Yano, K. and Kon, M., Structures on Manifolds, World Scientific, Singapore, 1984.

İstanbul University, Department of Mathematics, 34134, Vezneciler, İstanbul-TURKey

E-mail address: hakmete@istanbul.edu.tr

İstanbul University, Department of Mathematics, 34134, Vezneciler, İstanbul-TURKey

E-mail address: sibel.gerdan@istanbul.edu.tr 